QUARTERLY OF APPLIED MATHEMATICS

VOLUME LXX, NUMBER 1

MARCH 2012, PAGES 25-44

S 0033-569X(2011)01227-2

Article electronically published on August 26, 2011

\title{
GLOBAL WEAK SOLUTIONS TO THE EULER-BOLTZMANN EQUATIONS IN RADIATION HYDRODYNAMICS
}

\author{
BY \\ PENG JIANG (Department of Mathematics, Shanghai Jiao Tong University, Shanghai 200240, \\ People's Republic of China, and Department of Mathematics, University of Pittsburgh, Pittsburgh, \\ Pennsylvania 15260) \\ AND \\ DEHUA WANG (Department of Mathematics, University of Pittsburgh, Pittsburgh, Pennsylvania \\ 15260)
}

\begin{abstract}
The Cauchy problem for the one-dimensional Euler-Boltzmann equations in radiation hydrodynamics is studied. The global weak entropy solutions are constructed using the Godunov finite difference scheme. The global existence of weak entropy solutions in $L^{\infty}$ with arbitrarily large initial data is established with the aid of the compensated compactness method.
\end{abstract}

1. Introduction. In this paper we are interested in the global solution to the onedimensional Euler-Boltzmann equations in radiation hydrodynamics. Radiation hydrodynamics (cf. [17, 18]) is concerned with the propagation of thermal radiation and the effect of this radiation on the hydrodynamics describing the fluid motion.

The theory of radiation hydrodynamics finds a wide range of applications, including diverse astrophysical phenomena such as waves and oscillations in stellar atmospheres, nonlinear stellar pulsation, supernova explosions, stellar winds, and many other areas.

The mathematical equations governing the radiation hydrodynamics are the Euler equations of compressible fluids coupled with the Boltzmann equation of particle transport. It is very important to understand the solutions of the Euler-Boltzmann equations in order to obtain insights into the radiation hydrodynamics and related physical phenomena as well as applications. However, solving the Euler-Boltzmann equations is challenging because of the complexity and mathematical difficulty.

Received April 2, 2010.

2000 Mathematics Subject Classification. Primary 41A63, 78A40, 76N15, 76X05, 74G25, 54D30.

Key words and phrases. Radiation hydrodynamics, Euler equations, Boltzmann equation, global weak entropy solution, Godunov scheme, compensated compactness.

E-mail address: syepmathjp@yahoo.com.cn

E-mail address: dwang@math.pitt.edu

(C)2011 Brown University Reverts to public domain 28 years from publication 
We use $I(x, t, \nu, \Omega)$ to denote the specific intensity of radiation (at time $t$ ) at spatial point $x \in \mathbb{R}$, with frequency $\nu>0$ in a direction $\Omega \in S^{2}$. Then the system of partial differential equations of one-dimensional isentropic radiation hydrodynamics (cf. [17, 18]) consists of the following Boltzmann equation:

$$
\begin{aligned}
& \frac{1}{c} \frac{\partial I(\nu, \Omega)}{\partial t}+\Omega_{1} \frac{\partial I(\nu, \Omega)}{\partial x}=S(\nu)-\sigma_{a}(\nu, \rho) I(\nu, \Omega) \\
& \quad+\int_{0}^{\infty} d \nu^{\prime} \int_{S^{2}}\left(\frac{\nu}{\nu^{\prime}} \sigma_{s}\left(\nu^{\prime} \rightarrow \nu, \Omega^{\prime} \cdot \Omega, \rho\right) I\left(\nu^{\prime}, \Omega^{\prime}\right)-\sigma_{s}\left(\nu \rightarrow \nu^{\prime}, \Omega \cdot \Omega^{\prime}, \rho\right) I(\nu, \Omega)\right) d \Omega^{\prime}
\end{aligned}
$$

and the Euler equations:

$$
\left\{\begin{array}{l}
\rho_{t}+(\rho u)_{x}=0, \\
\left(\rho u+\frac{1}{c^{2}} F_{r}\right)_{t}+\left(\rho u^{2}+P_{r}+P_{m}\right)_{x}=0,
\end{array}\right.
$$

where $c$ denotes the light speed, $\Omega_{1}$ denotes the projection of $\Omega$ along the $x$-axis, $S(\nu)=$ $S(x, t, \nu, \Omega)$ denotes the rate of energy emission due to spontaneous processes, and $\sigma_{a}(\nu, \rho)=\sigma_{a}(x, t, \nu, \Omega, \rho)$ denotes the absorption coefficient. Similar to absorption, a photon can undergo scattering interactions with matter, and the scattering interactions change the photon's characteristics $\nu^{\prime}$ and $\Omega^{\prime}$ to a new set of characteristics $\nu$ and $\Omega$, which leads to the definition of the "differential scattering coefficient" $\sigma_{s}\left(\nu^{\prime} \rightarrow \nu, \Omega^{\prime} \cdot \Omega, \rho\right)=$ $\sigma_{s}\left(x, t, \nu^{\prime} \rightarrow \nu, \Omega^{\prime} \cdot \Omega, \rho\right)$. In the Euler equations (1.2),$\rho=\rho(x, t)$ is the density, $u=u(x, t)$ is the velocity, $P_{m}=P_{m}(\rho)$ is the pressure, $F_{r}$ and $P_{r}$ represent the radiative flux and the radiative pressure respectively defined by

$$
\left\{\begin{array}{l}
F_{r}=\int_{0}^{\infty} d \nu \int_{S^{2}} \Omega_{1} I(\nu, \Omega) d \Omega, \\
P_{r}=\frac{1}{c} \int_{0}^{\infty} d \nu \int_{S^{2}} \Omega_{1}^{2} I(\nu, \Omega) d \Omega .
\end{array}\right.
$$

In this paper we consider only polytropic ideal gases, in particular,

$$
P_{m}(\rho)=\rho^{\gamma} / \gamma,
$$

with $\gamma>1$ being the adiabatic index.

The existence of global weak entropy solutions in $L^{\infty}$ for the one-dimensional compressible Euler equations and some related applications has been established; see [2, 6, 7 ] and $[3,4,5,16,19,20]$ as well as the references therein. Due to its complexity, there are few mathematical results on the radiation hydrodynamical system (1.1)-(1.4). For the local existence of $C^{1}$ solutions and finite-time formation of singularities in solutions to the system of radiation hydrodynamics (1.1)-(1.4), see [10, 11]. For the studies of some simplified systems of radiation hydrodynamic models, see [1, 12, 13]. In this paper, we study the initial-value problem of (1.1)-(1.4) and establish the existence of global weak entropy solutions in $L^{\infty}$ with arbitrarily large data. We will construct the approximate solutions using the Godunov type finite scheme and apply the compensated compactness framework to prove the convergence of the approximate solutions. In particular, we show the $L^{\infty}$ uniform estimates, the $H^{-1}$ compactness, and the entropy conditions. To obtain the global existence, we need to overcome the complex structure of the system, especially from the radiation terms in the Euler equations (1.2), which requires some new ideas and new ingredients in the proof. 
The rest of this paper is organized as follows. In Section 2, we reformulate the problem and state the main result. In Section 3, we prove the main result, that is, the global existence of $L^{\infty}$ solutions, which consists of the construction of approximate solutions, convergence, and consistency as well as entropy conditions.

2. Reformulation and main result. Using (1.1) and (1.3), we can rewrite (1.1)(1.2) as

$$
\left\{\begin{array}{l}
\frac{1}{c} I_{t}+\Omega_{1} I_{x}=F(\rho, I) \\
\rho_{t}+m_{x}=0 \\
m_{t}+\left(\frac{m^{2}}{\rho}+P_{m}(\rho)\right)_{x}=G(\rho, I)
\end{array}\right.
$$

where $m=\rho u$ and

$$
\begin{aligned}
F(\rho, I)=S(\nu) & -\sigma(\nu, \rho) I(\nu, \Omega)+\int_{0}^{\infty} d \nu^{\prime} \int_{S^{2}}\left(\frac{\nu}{\nu^{\prime}} \sigma_{s}\left(\nu^{\prime} \rightarrow \nu, \Omega^{\prime} \cdot \Omega, \rho\right) I\left(\nu^{\prime}, \Omega^{\prime}\right)\right) d \Omega^{\prime} \\
G(\rho, I)=-\frac{1}{c} & \left(\int_{0}^{\infty} d \nu \int_{S^{2}} \Omega_{1}(S(\nu)-\sigma(\nu, \rho) I(\nu, \Omega)) d \Omega\right. \\
& \left.+\int_{0}^{\infty} d \nu \int_{S^{2}} d \Omega \int_{0}^{\infty} d \nu^{\prime} \int_{S^{2}} \Omega_{1} \frac{\nu}{\nu^{\prime}} \sigma_{s}\left(\nu^{\prime} \rightarrow \nu, \Omega^{\prime} \cdot \Omega, \rho\right) I\left(\nu^{\prime}, \Omega^{\prime}\right) d \Omega^{\prime}\right)
\end{aligned}
$$

with

$$
\sigma(\nu, \rho)=\sigma_{a}(\nu, \rho)+\int_{0}^{\infty} \int_{S^{2}} \sigma_{s}\left(\nu^{\prime} \rightarrow \nu, \Omega^{\prime} \cdot \Omega, \rho\right) d \Omega^{\prime} d \nu^{\prime} .
$$

We consider the initial-value problem of system (2.1) with the following initial data:

$$
\left\{\begin{array}{l}
\left.I\right|_{t=0}=I_{0}(x, \nu, \Omega), \quad x \in \mathbb{R}, \quad \nu>0, \quad \Omega \in S^{2}, \\
\left.\rho\right|_{t=0}=\rho_{0}(x),\left.\quad m\right|_{t=0}=m_{0}(x), \quad x \in \mathbb{R} .
\end{array}\right.
$$

We set $V:=(\rho, m)$ and $R_{T}:=\mathbb{R} \times[0, T]$.

Definition 2.1. The bounded measurable function $(I, V)=(I(x, t, \nu, \Omega), \rho(x, t)$, $m(x, t)), x \in \mathbb{R}, t>0$ is a global weak solution of (2.1)-(2.2) if the following integral identities hold for any fixed $T>0, \nu>0, \Omega \in S^{2}$ :

$$
\begin{gathered}
\int_{0}^{T} \int_{\mathbb{R}}\left(\frac{1}{c} I \varphi_{t}+\Omega_{1} I \varphi_{x}\right) d x d t+\int_{\mathbb{R}} \frac{1}{c} I_{0} \varphi(x, 0) d x+\int_{0}^{T} \int_{\mathbb{R}} F(\rho, I) \varphi d x d t=0, \\
\int_{0}^{T} \int_{\mathbb{R}}\left(\rho \varphi_{t}+m \varphi_{x}\right) d x d t+\int_{\mathbb{R}} \rho_{0} \varphi(x, 0) d x=0 \\
\int_{0}^{T} \int_{\mathbb{R}}\left(m \varphi_{t}+\left(\frac{m^{2}}{\rho}+P_{m}(\rho)\right) \varphi_{x}\right) d x d t+\int_{0}^{T} \int_{\mathbb{R}} G(\rho, I) \varphi d x+\int_{\mathbb{R}} m_{0} \varphi(x, 0) d x=0,
\end{gathered}
$$

for all $\varphi \in C_{0}^{\infty}\left(R_{T}\right)$ satisfying $\varphi(x, T)=0$ for $x \in \mathbb{R}$.

The main assumptions of this paper are the following:

(MA) Suppose that the initial data $\left(I_{0}, \rho_{0}, m_{0}\right)$ satisfies the conditions:

$$
\left|I_{0}(x, \nu, \Omega)\right| \leq M_{1}, \quad 0 \leq \rho_{0}(x) \leq M_{2}, \quad\left|m_{0}(x)\right| \leq M_{3} \rho_{0}(x),
$$


for some positive constants $M_{i}, i=1,2,3$, and suppose $F(\rho, I), G(\rho, I)$ satisfy the following conditions:

$$
\begin{gathered}
|F(\rho, I)| \leq C_{1}|I|+C_{2}\left|\rho^{\theta}\right|, \\
\left|\frac{G}{\rho}\right| \leq C_{3}|I|+C_{4}\left|\rho^{\theta}\right|,
\end{gathered}
$$

for some positive constants $C_{i}, i=1, \ldots, 4$, and for bounded $\rho_{i}, I_{i}, i=1,2$,

$$
\begin{aligned}
& \left\{\begin{array}{l}
\left|F\left(\rho_{1}, I_{1}\right)-F\left(\rho_{2}, I_{2}\right)\right| \leq C\left(I_{1}, I_{2}\right)\left|\rho_{1}-\rho_{2}\right|, \quad \text { if } \quad \rho_{1} \neq \rho_{2} \\
\left|F\left(\rho_{1}, I_{1}\right)-F\left(\rho_{2}, I_{2}\right)\right| \leq C\left|I_{1}-I_{2}\right|, \quad \text { if } \quad \rho_{1}=\rho_{2},
\end{array}\right. \\
& \left\{\begin{array}{l}
\left|G\left(\rho_{1}, I_{1}\right)-G\left(\rho_{2}, I_{2}\right)\right| \leq C\left(I_{1}, I_{2}\right)\left|\rho_{1}-\rho_{2}\right|, \quad \text { if } \quad \rho_{1} \neq \rho_{2} \\
\left|G\left(\rho_{1}, I_{1}\right)-G\left(\rho_{2}, I_{2}\right)\right| \leq C\left|I_{1}-I_{2}\right|, \quad \text { if } \quad \rho_{1}=\rho_{2},
\end{array}\right.
\end{aligned}
$$

where $C$ denotes a positive constant, $C\left(I_{1}, I_{2}\right)$ denotes a positive constant $C$ depending on $I_{1}$ and $I_{2}$, and $\theta=\frac{\gamma-1}{2}$. These assumptions hold for some class of functions $\sigma$ (in terms of $\left.\sigma_{a}, \sigma_{s}\right)$ and are required for the approach of compensated compactness in this paper. In particular, for $\sigma$ satisfying the following Lipschitz conditions (cf. [11]):

$$
\begin{gathered}
\left|\sigma\left(\rho_{1}\right)-\sigma\left(\rho_{2}\right)\right| \leq C\left|\rho_{1}-\rho_{2}\right| \\
\int_{0}^{\infty} d \nu^{\prime} \int_{S^{2}}\left|\frac{\nu}{\nu^{\prime}}\left(\sigma\left(\rho_{1}\right)-\sigma\left(\rho_{2}\right)\right)\right| d \Omega^{\prime} \leq C\left|\rho_{1}-\rho_{2}\right|,
\end{gathered}
$$

one has

$$
\begin{aligned}
\mid F\left(\rho_{1}, I_{1}\right)- & F\left(\rho_{2}, I_{2}\right)|\leq| \sigma\left(\rho_{1}\right) I_{1}-\sigma\left(\rho_{2}\right) I_{2}\left|+\int_{0}^{\infty} d \nu^{\prime} \int_{S^{2}}\right| \frac{\nu}{\nu^{\prime}}\left(\sigma\left(\rho_{1}\right) I_{1}-\sigma\left(\rho_{2}\right) I_{2}\right) \mid d \Omega^{\prime} \\
\leq & C\left(I_{1}, I_{2}\right)\left|\sigma\left(\rho_{1}\right)-\sigma\left(\rho_{2}\right)\right|+C\left(I_{1}, I_{2}\right) \int_{0}^{\infty} d \nu^{\prime} \int_{S^{2}}\left|\frac{\nu}{\nu^{\prime}}\left(\sigma\left(\rho_{1}\right)-\sigma\left(\rho_{2}\right)\right)\right| d \Omega^{\prime} \\
\leq & C\left(I_{1}, I_{2}\right)\left|\rho_{1}-\rho_{2}\right| .
\end{aligned}
$$

Similarly, (2.9) holds. Now we state the main result of this paper as follows.

Theorem 2.1. Under the above assumption (MA), for $1<\gamma \leq 2$, the Cauchy problem (2.1)-(2.2) has a global weak entropy solution $(I(x, t, \nu, \Omega), \rho(x, t), m(x, t)), x \in \mathbb{R}, t>0$, for any fixed $\nu>0, \Omega \in S^{2}$, satisfying the following estimates and entropy condition: on $R_{T}=\mathbb{R} \times[0, T]$ for any fixed $T>0$,

$$
|I| \leq C(T), \quad 0 \leq \rho \leq C(T), \quad|m| \leq C(T) \rho,
$$

for a constant $C(T)>0$ which depends on $T$, and

$$
\int_{0}^{T} \int_{\mathbb{R}}\left(\eta(\rho, m) \psi_{t}+q(\rho, m) \psi_{x}\right) d x d t+\int_{0}^{T} \int_{\mathbb{R}} \eta_{m}(\rho, m) G(\rho, I) \psi d x d t \geq 0
$$

for all weak and convex entropy pairs $(\eta, q)$ of the Euler equations in (2.1) and for all nonnegative test functions $\psi \in C_{0}^{\infty}\left(R_{T}\right)$, with $\psi(x, 0)=\psi(x, T)=0$. 
3. Proof of the main result: Theorem 2.1, In this section, we prove our main result in Theorem 2.1. We divide the proof of the theorem into three steps. The first step is to construct the approximate solutions by the fractional Godunov schemes, the second step is to show the convergence by the compensated compactness framework, and the final step is to prove that the limit function is a weak entropy solution. We recall that the homogeneous system:

$$
\left\{\begin{array}{l}
\rho_{t}+m_{x}=0 \\
m_{t}+\left(\frac{m^{2}}{\rho}+P_{m}(\rho)\right)_{x}=0
\end{array}\right.
$$

has two eigenvalues

$$
\lambda_{1}=\frac{m}{\rho}-\rho^{\theta}, \quad \lambda_{2}=\frac{m}{\rho}+\rho^{\theta}
$$

and Riemann invariants

$$
w=\frac{m}{\rho}+\frac{\rho^{\theta}}{\theta}, \quad z=\frac{m}{\rho}-\frac{\rho^{\theta}}{\theta},
$$

with $\theta=\frac{\gamma-1}{2}$.

3.1. Construction of approximate solutions. In this section, we construct the approximate solutions $\left(I^{l}, V^{l}\right)=\left(I^{l}, \rho^{l}, m^{l}\right)$ with $V^{l}=\left(\rho^{l}, m^{l}\right)$ of $(2.1)-(2.2)$ in the strip $0 \leq t \leq T$ for any fixed $T>0$, where $l$ and $h$ are the space mesh length and the time mesh length, respectively, satisfying the Courant-Friedrichs-Lewy condition:

$$
\max _{i=1,2}\left(\sup _{x, t}\left|\lambda_{i}\left(\rho^{l}, m^{l}\right)\right|\right) \leq \frac{l}{2 h} .
$$

We now use the Godunov scheme to construct a sequence of approximate solutions of (2.1). Namely, we solve the Riemann problems of (3.1) in the region

$$
\begin{aligned}
& R_{j}^{1} \equiv\left\{(x, t): x_{j-1 / 2} \leq x \leq x_{j+1 / 2}, 0 \leq t<h\right\}, \quad x_{j \pm 1 / 2}=(j \pm 1 / 2) l: \\
& \left\{\begin{array}{l}
\left(\rho_{0}^{l}\right)_{t}+\left(m_{0}^{l}\right)_{x}=0, \\
\left(m_{0}^{l}\right)_{t}+\left(\frac{\left(m_{0}^{l}\right)^{2}}{\rho}+P_{m}\left(\rho_{0}^{l}\right)\right)_{x}=0, \\
\left.\left(\rho_{0}^{l}, m_{0}^{l}\right)\right|_{t=0}= \begin{cases}\frac{1}{l} \int_{(j-1) l}^{j l}\left(\rho_{0}(x), m_{0}(x)\right) d x, & x<j l, \\
\frac{1}{l} \int_{j l}^{(j+1) l}\left(\rho_{0}(x), m_{0}(x)\right) d x, & x>j l,\end{cases}
\end{array}\right.
\end{aligned}
$$

for all integers $j \geq 1$. We also solve the following problem in the strip: $x \in \mathbb{R}, 0 \leq t \leq h$,

$$
\left\{\begin{array}{l}
\frac{1}{c}\left(I_{0}^{l}\right)_{t}+\Omega_{1}\left(I_{0}^{l}\right)_{x}=0 \\
\left.I_{0}^{l}\right|_{t=0}=I_{0}(x)
\end{array}\right.
$$

Then, we set

$$
\left\{\begin{array}{l}
I^{l}(\nu, \Omega, x, t)=I_{0}^{l}(\nu, \Omega, x, t)+c F\left(\rho_{0}^{l}, I_{0}^{l}\right) t, \\
V^{l}(x, t)=V_{0}^{l}(x, t)+\widetilde{G}\left(\rho_{0}^{l}, I_{0}^{l}\right) t,
\end{array}\right.
$$

for $x \in \mathbb{R}, 0 \leq t \leq h$, where $V_{0}^{l}=\left(\rho_{0}^{l}, m_{0}^{l}\right)$ and $\widetilde{G}=(0, G)^{\top}$. We continue the construction to the next time step recursively as follows. 
Suppose that we have defined approximate solutions $\left(I^{l}, V^{l}\right)$ for $0 \leq t<i h$, with $i \geq 1$ an integer. We then define

$$
\left\{\begin{array}{l}
I^{l}(\nu, \Omega, x, t)=I_{0}^{l}(\nu, \Omega, x, t)+F\left(\rho_{0}^{l}, I_{0}^{l}\right)(t-i h), \\
V^{l}(x, t)=V_{0}^{l}(x, t)+\widetilde{G}\left(\rho_{0}^{l}, I_{0}^{l}\right)(t-i h),
\end{array}\right.
$$

for $i h \leq t<(i+1) h$, where $V_{0}^{l}(x, t)$ are piecewise smooth functions defined as a solution of the Riemann problem in the region $R_{j}^{i+1} \equiv\left\{(x, t): x_{j-1 / 2} \leq x \leq x_{j+1 / 2}\right.$, ih $\leq t<$ $(i+1) h\}$ :

$$
\left\{\begin{array}{l}
\left(\rho_{0}^{l}\right)_{t}+\left(m_{0}^{l}\right)_{x}=0, \\
\left(m_{0}^{l}\right)_{t}+\left(\frac{\left(m_{0}^{l}\right)^{2}}{\rho}+P_{m}\left(\rho_{0}^{l}\right)\right)_{x}=0, \\
\left.\left(\rho_{0}^{l}, m_{0}^{l}\right)\right|_{t=i h}= \begin{cases}\frac{1}{l} \int_{(j-1) l}^{j l}\left(\rho^{l}(x, i h-0), m^{l}(x, i h-0)\right) d x, & x<j l, \\
\frac{1}{l} \int_{j l}^{(j+1) l}\left(\rho^{l}(x, i h-0), m^{l}(x, i h-0)\right) d x, & x>j l,\end{cases}
\end{array}\right.
$$

and $I_{0}^{l}(\nu, \Omega, x, t)$ is the solution of following problem in the region $x \in \mathbb{R}, i h \leq t<(i+1) h$ :

$$
\left\{\begin{array}{l}
\frac{1}{c}\left(I_{0}^{l}\right)_{t}+\Omega_{1}\left(I_{0}^{l}\right)_{x}=0, \\
\left.I_{0}^{l}\right|_{t=i h}=I^{l}(\nu, \Omega, x, i h-0) .
\end{array}\right.
$$

Then the approximate solutions $\left(I^{l}(\nu, \Omega, x, t), V^{l}(x, t)\right)$ are well-defined for $x \in \mathbb{R}, 0 \leq$ $t \leq T$ and any fixed $\nu>0, \Omega \in S^{2}$.

3.2. Convergence via compensated compactness method. We now show the convergence of the approximate solutions constructed in Section 3.1 with the aid of the compensated compactness method. The following is the compensated compactness framework (cf. [2, 7, 14, 15]):

THEOREM 3.1. Suppose that a sequence of measurable functions $\left(V^{l}(x, t), I^{l}(\nu, \Omega, x, t)\right)=\left(\rho^{l}(x, t), m^{l}(x, t), I^{l}(\nu, \Omega, x, t)\right), x \in \mathbb{R}, t>0, \nu>0, \Omega \in S^{2}$, satisfies the following conditions, for each fixed pair of parameters $(\nu, \Omega)$.

(1) There is a constant $C>0$ such that, for $(x, t) \in \mathbb{R}_{+}^{2}:=\mathbb{R} \times(0, \infty)$,

$$
\left|I^{l}(\nu, \Omega, x, t)\right| \leq C, \quad 0 \leq \rho^{l}(x, t) \leq C, \quad\left|m^{l}(x, t)\right| \leq C \rho^{l}(x, t) .
$$

(2) The measure sequence

$$
\eta\left(V^{l}\right)_{t}+q\left(V^{l}\right)_{x}
$$

is compact in $H_{\text {loc }}^{-1}\left(\Omega^{\prime}\right)$, for all weak entropy pairs $(\eta, q)$ of $(3.1)$, where $\Omega^{\prime} \subset \mathbb{R}_{+}^{2}$ is any bounded and open set. Then, for $1<\gamma \leq 2$, there exist functions $(\rho, m, I) \in L^{\infty}\left(\mathbb{R}_{+}^{2}\right)$ and a convergent subsequence (still labeled) $\left(V^{l}, I^{l}\right)$, such that as $l \rightarrow 0, V^{l}(x, t) \rightarrow V(x, t)=$ $(\rho(x, t), m(x, t))$ almost everywhere in $\mathbb{R}_{+}^{2}$, and $I^{l}(\nu, \Omega, x, t) \rightarrow I(\nu, \Omega, x, t)$ weakly-* in $\mathbb{R}_{+}^{2}$ for each fixed pair of parameters $(\nu, \Omega)$.

We now prove that the approximate solutions $\left(V^{l}, I^{l}\right)$ satisfy the above framework, i.e., the $L^{\infty}$ estimate and the $H^{-1}$ estimate. 
3.2.1. $L^{\infty}$ estimate. First, we prove that the approximate solutions $\left(V^{l}, I^{l}\right)$ have a uniform bound.

TheOREm 3.2. Suppose that the initial data $\left(I_{0}, \rho_{0}, m_{0}\right)$ satisfy the following conditions:

$$
\left|I_{0}(x, \nu, \Omega)\right| \leq C_{0}, \quad 0 \leq \rho_{0}(x) \leq C_{0}, \quad\left|m_{0}(x)\right| \leq C_{0} \rho_{0}(x), \quad x \in \mathbb{R}, \nu>0, \quad \Omega \in S^{2},
$$

for some constant $C_{0}>0$, and $F(\rho, I), G(\rho, I)$ satisfy (2.6)-(2.7). Then, there exists a positive constant $C(T)$, independent of $l$ and $h$, such that

$$
\left|I^{l}\right| \leq C(T), \quad 0 \leq \rho^{l}(x, t) \leq C(T), \quad\left|m^{l}\right| \leq C(T) \rho^{l}, \quad(x, t) \in R_{T}=\mathbb{R} \times[0, T] .
$$

Proof. For $x \in \mathbb{R}, i h \leq t<(i+1) h$ with $i \geq 0$ integers,

$$
\begin{aligned}
\left|I^{l}(x, t)\right| \leq & \left\|I_{0}^{l}\right\|_{L^{\infty}}+\|F\|_{L^{\infty}}(t-i h) \\
\leq & \left\|I_{0}^{l}\right\|_{L^{\infty}}+C\left(\left\|I_{0}^{l}\right\|_{L^{\infty}}+\left\|\left(\rho_{0}^{l}\right)^{\theta}\right\|_{L^{\infty}}\right) h \\
\leq & \left\|I_{0}^{l}\right\|_{L^{\infty}}+C\left(\left\|I_{0}^{l}\right\|_{L^{\infty}}+\left\|w_{0}^{l}-z_{0}^{l}\right\|_{L^{\infty}}\right) h \\
\leq & \left\|I_{0}^{l}(\nu, \Omega, x, i h+0)\right\|_{L^{\infty}} \\
& +C\left(\left\|I_{0}^{l}(\nu, \Omega, x, i h+0)\right\|_{L^{\infty}}+\sup _{x} w_{0}^{l}(x, i h+0)-\inf _{x} z_{0}^{l}(x, i h+0)\right) h,
\end{aligned}
$$

where $w_{0}^{l}$ and $z_{0}^{l}$ are Riemann invariants corresponding to the Riemann solutions $V_{0}^{l}$. For the Riemann invariants, we have the following estimates:

$$
\begin{aligned}
w^{l}(x, t)= & w_{0}^{l}(x, t)+\frac{G}{\rho_{0}^{l}}(t-i h) \\
\leq & \sup _{x} w_{0}^{l}(x, i h+0)+\left\|\frac{G}{\rho_{0}^{l}}\right\|_{L^{\infty}} h \\
\leq & \sup _{x} w_{0}^{l}(x, i h+0)+C\left(\left\|I_{0}^{l}\right\|_{L^{\infty}}+\left\|w_{0}^{l}-z_{0}^{l}\right\|_{L^{\infty}}\right) h \\
\leq & \sup _{x} w_{0}^{l}(x, i h+0) \\
& +C\left(\left\|I_{0}^{l}(\nu, \Omega, x, i h+0)\right\|_{L^{\infty}}+\sup _{x} w_{0}^{l}(x, i h+0)-\inf _{x} z_{0}^{l}(x, i h+0)\right) h
\end{aligned}
$$

and

$$
\begin{aligned}
z^{l}(x, t)= & z_{0}^{l}(x, t)+\frac{G}{\rho_{0}^{l}}(t-i h) \\
\geq & \inf _{x} z_{0}^{l}(x, i h+0)+\left\|\frac{G}{\rho_{0}^{l}}\right\|_{L^{\infty}} h \\
\geq & \inf _{x} z_{0}^{l}(x, i h+0)-C\left(\left\|I_{0}^{l}\right\|_{L^{\infty}}+\left\|w_{0}^{l}-z_{0}^{l}\right\|_{L^{\infty}}\right) h \\
\geq & \inf _{x} z_{0}^{l}(x, i h+0) \\
& -C\left(\left\|I_{0}^{l}(\nu, \Omega, x, i h+0)\right\|_{L^{\infty}}+\sup _{x} w_{0}^{l}(x, i h+0)-\inf _{x} z_{0}^{l}(x, i h+0)\right) h .
\end{aligned}
$$

Let

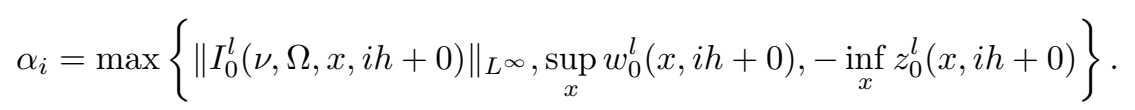


Then

$$
\begin{aligned}
& \max \left\{\left\|I_{0}^{l}(\nu, \Omega, x,(i+1) h-0)\right\|_{L^{\infty}}, \sup _{x} w^{l}(x,(i+1) h-0),-\inf _{x} z_{0}^{l}(x,(i+1) h-0)\right\} \\
& \leq C \alpha_{i}(1+h) .
\end{aligned}
$$

It follows that

$$
\alpha_{i+1} \leq C(1+h) \alpha_{i}
$$

Thus

$$
\alpha_{i+1} \leq C(1+h) \alpha_{i} \leq C(1+h)^{i+1} \alpha_{0}, \quad 0 \leq i \leq n=\frac{T}{h} .
$$

We can deduce that

$$
\alpha_{i} \leq C(1+h)^{i} \alpha_{0} \leq C(1+h)^{n} \alpha_{0}=C(1+h)^{\frac{T}{h}} \alpha_{0} \leq C e^{T} \alpha_{0},
$$

where $\alpha_{0}=\max \left\{\left\|I_{0}\right\|_{L^{\infty}}, \sup _{x} w_{0}(x),-\inf _{x} z_{0}(x)\right\}$. Then

$$
w^{l}(x, t) \leq C e^{T} \alpha_{0}, \quad z^{l}(x, t) \leq C e^{T} \alpha_{0}, \quad w^{l}-z^{l} \geq 0 .
$$

Then, there is a constant $C(T)>0$ independent of $l$ and $h$ such that

$$
\left|I^{l}(\nu, \Omega, x, t)\right| \leq C(T), \quad 0 \leq \rho^{l}(x, t) \leq C(T), \quad\left|m^{l}(x, t)\right| \leq C(T) \rho^{l}(x, t) .
$$

Now, we can choose the time mesh length $h=h(l)$. Let

$$
\lambda=\max _{i=1,2}\left\{\sup _{0 \leq \rho \leq C(T),|m| \leq C(T) \rho}\left|\lambda_{i}(\rho, m)\right|\right\},
$$

and take $h=\frac{T}{n}$, where $n=\left[\frac{4 \lambda T}{l}\right]+1$. Then the Courant-Friedrichs-Lewy condition holds.

3.2.2. $H^{-1}$ estimate. In order to prove the $H^{-1}$ estimate, we need some useful lemmas. Lemma 3.1. Let $V^{l}(x, t)$ be the approximate solution. Then, there is a positive constant $C$ independent of $l$ such that

$$
\sum_{i, j} \int_{(j-1) l}^{j l}\left|V(x, i h-0)-V_{j}^{i}\right|^{2} d x \leq C
$$

where

$$
V_{j}^{i}=\frac{1}{l} \int_{(j-1) l}^{j l} V^{l}(x, i h-0) d x .
$$

Proof. Consider the mechanical entropy pair

$$
\eta_{*}=\frac{1}{2} \frac{m^{2}}{\rho}+\frac{\rho^{\gamma}}{\gamma(\gamma-1)}, \quad q_{*}=\frac{1}{2} \frac{m^{3}}{\rho^{2}}+\frac{1}{\gamma-1} \rho^{\gamma-1} m .
$$

For $i h \leq t \leq(i+1) h$, Green's formula implies that

$$
\begin{aligned}
& \sum_{i, j} \int_{(j-1) l}^{j l}\left(\eta_{*}\left(V_{0}^{l}\right)^{\underline{i}}-\eta_{*}\left(V_{j}^{i}\right)\right) d x+\int_{0}^{T} \sum\left\{\sigma\left[\eta_{*}\right]-\left[q_{*}\right]\right\} d t \\
& =\int_{\Omega^{\prime}}\left(\eta_{*}\left(V_{0}^{l}(x, 0)\right)-\eta_{*}\left(V_{0}^{l}(x, T-0)\right)\right) d x
\end{aligned}
$$


where $\left(V_{0}^{l}\right)^{\underline{i}}=V_{0}^{l}(x, i h-0), \sigma$ is the propagating speed of the shock wave, and

$$
\begin{gathered}
{\left[\eta_{*}\right]=\eta_{*}\left(V_{0}^{l}(x(t)+0, t)\right)-\eta_{*}\left(V_{0}^{l}(x(t)-0, t)\right),} \\
{\left[q_{*}\right]=q_{*}\left(V_{0}^{l}(x(t)+0, t)\right)-q_{*}\left(V_{0}^{l}(x(t)-0, t)\right), \quad i h \leq t<(i+1) h .}
\end{gathered}
$$

From the uniform bound of $V_{0}^{l}$, the right-hand side of (3.7) is dominated by a constant $C>0$ independent of $l$; i.e.,

$$
\sum_{i, j} \int_{(j-1) l}^{j l}\left(\eta_{*}\left(V_{0}^{l}\right)^{\underline{i}}-\eta_{*}\left(V_{j}^{i}\right)\right) d x+\int_{0}^{T} \sum\left\{\sigma\left[\eta_{*}\right]-\left[q_{*}\right]\right\} d t \leq C .
$$

We decompose the first term of (3.8) into two parts:

$$
\begin{aligned}
& \int_{(j-1) l}^{j l}\left(\eta_{*}\left(V_{0}^{l}\right)^{\underline{i}}-\eta_{*}\left(V_{j}^{i}\right)\right) d x \\
& =\int_{(j-1) l}^{j l}\left(\eta_{*}\left(V^{l}\right)^{\underline{i}}-\eta_{*}\left(V_{j}^{i}\right)\right) d x+\int_{(j-1) l}^{j l}\left(\eta_{*}\left(V_{0}^{l}\right)^{\underline{i}}-\eta_{*}\left(V^{l}\right)^{\underline{i}}\right) d x \\
& \equiv A_{j}^{i}+R_{j}^{i},
\end{aligned}
$$

where $\left(V^{l}\right)^{\underline{i}}=V^{l}(x, i h-0)$. For $A_{j}^{i}$, we take the Taylor expansion for $\eta_{*}\left(V^{l}\right)^{\underline{i}}$ to get

$$
\eta_{*}\left(V^{l}\right)^{\underline{i}}=\eta_{*}\left(V_{j}^{i}\right)+\nabla \eta_{*}\left(V_{j}^{i}\right)\left(\left(V^{l}\right)^{\underline{i}}-V_{j}^{i}\right)+\frac{1}{2}\left(\left(V^{l}\right)^{\underline{i}}-V_{j}^{i}\right)^{\top} \nabla^{2} \eta_{*}\left(\xi_{j}^{i}\right)\left(\left(V^{l}\right)^{\underline{i}}-V_{j}^{i}\right),
$$

where $\xi_{j}^{i}$ is a mean value. Integrating $(3.9)$ on the cell $((j-1) l, j l)$ and using the fact that $V_{j}^{i}$ is the average value of $\left(V^{l}\right)^{i}$ on this cell, we have

$$
A_{j}^{i}=\frac{1}{2} \int_{(j-1) l}^{j l}\left(\left(V^{l}\right)^{\underline{i}}-V_{j}^{i}\right)^{\top} \nabla^{2} \eta_{*}\left(\xi_{j}^{i}\right)\left(\left(V^{l}\right)^{\underline{i}}-V_{j}^{i}\right) d x .
$$

For $R_{j}^{i}$, we have

$$
\begin{aligned}
R_{j}^{i} & =\int_{(j-1) l}^{j l}\left(\int_{0}^{1} \nabla \eta_{*}\left(\left(V^{l}\right)^{\underline{i}}+\theta\left(\left(V_{0}^{l}\right)^{\underline{i}}-\left(V^{l}\right)^{\underline{i}}\right)\right) d \theta\left(\left(V_{0}^{l}\right)^{\underline{i}}-\left(V^{l}\right)^{\underline{\underline{i}}}\right)\right) d x \\
& =-h \int_{(j-1) l}^{j l}\left(\int_{0}^{1} \nabla \eta_{*}\left(\left(V^{l}\right)^{\underline{\underline{i}}}+\theta\left(\left(V_{0}^{l}\right)^{\underline{\underline{i}}}-\left(V^{l}\right)^{\underline{\underline{i}}}\right)\right) d \theta G\left(\left(\rho_{0}^{l}\right)^{\underline{i}},\left(I_{0}^{l}\right)^{\underline{\underline{i}}}\right)\right) d x .
\end{aligned}
$$

From the uniform bound of approximate solutions, we get $\left|R_{j}^{i}\right| \leq C l h$, where $C$ is independent of $l$ and $h$.

Summing over all cells, by (3.8), (3.10) and (3.11), we have

$$
\sum_{i, j} \int_{(j-1) l}^{j l}\left(\left(V^{l}\right)^{\underline{i}}-V_{j}^{i}\right)^{\top} \nabla^{2} \eta_{*}\left(\xi_{j}^{i}\right)\left(\left(V^{l}\right)^{\underline{i}}-V_{j}^{i}\right) d x+2 \int_{0}^{T} \sum\left\{\sigma\left[\eta_{*}\right]-\left[q_{*}\right]\right\} d t \leq C .
$$

Since $\left(\eta_{*}, q_{*}\right)$ is a convex entropy pair, $\sigma\left[\eta_{*}\right]-\left[q_{*}\right] \geq 0$ holds across the shock waves. Thus

$$
\int_{0}^{T} \sum\left\{\sigma\left[\eta_{*}\right]-\left[q_{*}\right]\right\} d t \leq C
$$

and

$$
\sum_{i, j} \int_{(j-1) l}^{j l}\left(\left(V^{l}\right)^{\underline{i}}-V_{j}^{i}\right)^{\top} \nabla^{2} \eta_{*}\left(\xi_{j}^{i}\right)\left(\left(V^{l}\right)^{\underline{i}}-V_{j}^{i}\right) d x \leq C .
$$


In particular, $\eta_{*}$ is strictly convex; i.e., there is a constant $\alpha>0$ such that

$$
V^{\top} \nabla^{2} \eta_{*} V \geq \alpha|V|^{2} .
$$

It follows from (3.12) that (3.6) is true.

The proofs of the following three lemmas can be found in $[6,8$.

Lemma 3.2. Assume that $0 \leq \rho \leq C,|m| \leq C \rho$. Then, there is a constant $C^{\prime}>0$ such that

$$
|\nabla \eta| \leq C^{\prime}, \quad|\nabla q| \leq C^{\prime}, \quad\left|V^{\top} \nabla^{2} \eta V\right| \leq C^{\prime} V^{\top} \nabla^{2} \eta_{*} V
$$

for every weak entropy pair $(\eta, q)$ of $(3.1)$.

Lemma 3.3. For every weak entropy pair $(\eta, q)$ of $(3.1)$, there is a constant $C>0$ such that

$$
|\sigma[\eta]-[q]| \leq C\left\{\sigma\left[\eta_{*}\right]-\left[q_{*}\right]\right\} .
$$

Lemma 3.4. Let $\Omega \subset \mathbb{R}^{N}$ be a bounded open set. Then,

(compact set of $\left.W^{-1, p}(\Omega)\right) \cap\left(\right.$ bounded set of $\left.W^{-1, r}(\Omega)\right) \subset\left(\right.$ compact set of $H_{l o c}^{-1}(\Omega)$ ), for some constants $p$ and $r$ satisfying $1<p \leq 2<r<\infty$.

Now, we can prove that the sequence of entropy dissipation measures $\eta\left(V^{l}\right)_{t}+q\left(V^{l}\right)_{x}$ is compact in $H_{l o c}^{-1}$.

Theorem 3.3. For the approximate solution $\left\{V^{l}\right\}$, the measure sequence

$$
\eta\left(V^{l}\right)_{t}+q\left(V^{l}\right)_{x} \text { is a compact subset of } H_{l o c}^{-1}\left(\Omega^{\prime}\right)
$$

for all weak entropy pairs $(\eta, q)$, where $\Omega^{\prime}$ is any open bounded subset in $R_{T}=\mathbb{R} \times[0, T]$.

Proof. For any $\varphi \in C_{0}^{\infty}\left(\Omega^{\prime}\right)$, we consider

$$
\int_{0}^{T} \int_{\Omega^{\prime}}\left(\eta\left(V^{l}\right) \varphi_{t}+q\left(V^{l}\right) \varphi_{x}\right) d x d t=A(\varphi)+R(\varphi)+B(\varphi)+\Sigma(\varphi)+S(\varphi),
$$

where

$$
\begin{gathered}
A(\varphi)=\sum_{i, j} \int_{(j-1) l}^{j l}\left(\eta\left(V^{l}\right)^{\underline{i}}-\eta\left(V_{j}^{i}\right)\right) \varphi(x, i h) d x, \\
R(\varphi)=\sum_{i, j} \int_{(j-1) l}^{j l}\left(\eta\left(V_{0}^{l}\right)^{\underline{i}}-\eta\left(V^{l}\right)^{\underline{i}}\right) \varphi(x, i h) d x, \\
B(\varphi)=\int_{\Omega^{\prime}}\left(\eta\left(V_{0}^{l}(x, T)\right) \varphi(x, T)-\eta\left(V_{0}^{l}(x, 0)\right) \varphi(x, 0)\right) d x, \\
\Sigma(\varphi)=\int_{0}^{T} \sum\{\sigma[\eta]-[q]\} \varphi(x(t), t) d t, \\
S(\varphi)=\int_{0}^{T} \int_{\Omega^{\prime}}\left(\left(\eta\left(V^{l}\right)-\eta\left(V_{0}^{l}\right)\right) \varphi_{t}+\left(q\left(V^{l}\right)-q\left(V_{0}^{l}\right)\right) \varphi_{x}\right) d x d t .
\end{gathered}
$$

We now make estimates for each of these terms. 
(1) We decompose $A(\varphi)$ into two parts:

$$
\begin{aligned}
A(\varphi) & =\sum_{i, j} \varphi_{j}^{i} \int_{(j-1) l}^{j l}\left(\eta\left(V^{l}\right)^{\underline{i}}-\eta\left(V_{j}^{i}\right)\right) d x+\sum_{i, j} \int_{(j-1) l}^{j l}\left(\eta\left(V^{l}\right)^{\underline{i}}-\eta\left(V_{j}^{i}\right)\right)\left(\varphi^{i}-\varphi_{j}^{i}\right) d x \\
& \equiv A_{1}(\varphi)+A_{2}(\varphi),
\end{aligned}
$$

where $\varphi_{j}^{i}=\varphi(j l, i h)$ and $\varphi^{i}=\varphi(x, i h)$. For $A_{1}(\varphi)$, using (3.9), (3.10), (3.12) and Lemma 3.2 we have

$$
\begin{aligned}
\left|A_{1}(\varphi)\right| & =\frac{1}{2}\left|\sum_{i, j} \varphi_{j}^{i} \int_{(j-1) l}^{j l}\left(\left(V^{l}\right)^{\underline{i}}-V_{j}^{i}\right)^{\top} \nabla^{2} \eta\left(\xi_{j}^{i}\right)\left(\left(V^{l}\right)^{\underline{i}}-V_{j}^{i}\right) d x\right| \\
& \leq C\|\varphi\|_{L^{\infty}} \sum_{i, j} \int_{(j-1) l}^{j l}\left(\left(V^{l}\right)^{\underline{i}}-V_{j}^{i}\right)^{\top} \nabla^{2} \eta_{*}\left(\xi_{j}^{i}\right)\left(\left(V^{l}\right)^{\underline{i}}-V_{j}^{i}\right) d x \\
& \leq C\|\varphi\|_{L^{\infty}} .
\end{aligned}
$$

For $A_{2}(\varphi)$, using Lemma 3.1 and Lemma 3.2 , we have

$$
\begin{aligned}
\left|A_{2}(\varphi)\right| & \leq\left(\sum_{i, j} \int_{(j-1) l}^{j l}\left(\varphi^{i}-\varphi_{j}^{i}\right)^{2} d x\right)^{\frac{1}{2}}\left(\sum_{i, j} \int_{(j-1) l}^{j l}\left(\eta\left(V^{l}\right)^{\underline{i}}-\eta\left(V_{j}^{i}\right)\right)^{2} d x\right)^{\frac{1}{2}} \\
& \leq C\|\varphi\|_{C_{0}^{\alpha}}\left(\sum_{i, j} \int_{(j-1) l}^{j l}|x-j l|^{2 \alpha} d x\right)^{\frac{1}{2}}\left(\sum_{i, j} \int_{(j-1) l}^{j l}\left|\nabla \eta\left(\left(V^{l}\right)^{\underline{i}}-V_{j}^{i}\right)\right|^{2} d x\right)^{\frac{1}{2}} \\
& \leq C l^{\alpha-1 / 2}\|\varphi\|_{C_{0}^{\alpha}}\|\nabla \eta\|_{L^{\infty}}\left(\sum_{i, j} \int_{(j-1) l}^{j l}\left(\left(V^{l}\right)^{\underline{i}}-V_{j}^{i}\right)^{2} d x\right)^{\frac{1}{2}} \\
& \leq C l^{\alpha-1 / 2}\|\varphi\|_{C_{0}^{\alpha}},
\end{aligned}
$$

where $\frac{1}{2}<\alpha<1$.

(2) For $R(\varphi)$, with the help of Theorem 3.2 and Lemma 3.2 we deduce that

$$
\begin{aligned}
|R(\varphi)| & \leq \sum_{i, j} \int_{(j-1) l}^{j l}\left|\nabla \eta\left(\xi_{j}^{i}\right)\left(\left(V_{0}^{l}\right)^{\underline{i}}-\left(V^{l}\right)^{\underline{i}}\right) \varphi^{i}\right| d x \\
& \leq h\|\nabla \eta\|_{L^{\infty}}\|\varphi\|_{L^{\infty}} \sum_{i, j} \int_{(j-1) l}^{j l}\left|G\left(\left(\rho_{0}^{l}\right)^{\underline{i}},\left(I_{0}^{l}\right)^{\underline{i}}\right)\right| d x \\
& \leq C h\|\nabla \eta\|_{L^{\infty}}\|\varphi\|_{L^{\infty}}\left\|G\left(\left(\rho_{0}^{l}\right)^{\underline{i}},\left(I_{0}^{l}\right)^{\underline{i}}\right)\right\|_{L^{\infty} n} \\
& \leq C\|\varphi\|_{L^{\infty}} .
\end{aligned}
$$

(3) For $B(\varphi)$, we have

$$
\begin{aligned}
|B(\varphi)| & \leq\|\varphi\|_{L^{\infty}} \int_{\Omega^{\prime}}\left(\left|\eta\left(V_{0}^{l}(x, T)\right)\right|+\left|\eta\left(V_{0}^{l}(x, 0)\right)\right|\right) d x \\
& \leq C\|\varphi\|_{L^{\infty}} .
\end{aligned}
$$


(4) It follows from Lemma 3.3 that

$$
\begin{aligned}
|\Sigma(\varphi)| & \leq\|\varphi\|_{L^{\infty}} \int_{0}^{T} \sum|\sigma[\eta]-[q]| d t \\
& \leq C\|\varphi\|_{L^{\infty}} \int_{0}^{T} \sum\left\{\sigma\left[\eta_{*}\right]-\left[q_{*}\right]\right\} d t \\
& \leq C\|\varphi\|_{L^{\infty}} .
\end{aligned}
$$

(5) Using Lemma 3.2 we have

$$
\begin{aligned}
|S(\varphi)| & \leq \int_{0}^{T} \int_{\Omega^{\prime}}\left(\left|\nabla \eta\left(\xi_{1}\right)\right|\left|\varphi_{t}\right|+\left|\nabla q\left(\xi_{2}\right) \| \varphi_{x}\right|\right)\left|V^{l}-V_{0}^{l}\right| d x d t \\
& \leq h\left\|G\left(\rho_{0}^{l}, I_{0}^{l}\right)\right\|_{L^{\infty}}\left(\|\nabla \eta\|_{L^{\infty}}+\|\nabla q\|_{L^{\infty}}\right) \int_{0}^{T} \int_{\Omega^{\prime}}\left(\left|\varphi_{t}\right|+\left|\varphi_{x}\right|\right) d x d t \\
& \leq C l\|\varphi\|_{H_{0}^{1}\left(\Omega^{\prime}\right)} .
\end{aligned}
$$

Since $C_{0}^{\infty}\left(\Omega^{\prime}\right)$ is dense in $H_{0}^{1}\left(\Omega^{\prime}\right)$, it follows that

$$
\|S\|_{H_{l o c}^{-1}\left(\Omega^{\prime}\right)} \leq C l \rightarrow 0 \quad \text { as } \quad l \rightarrow 0 .
$$

Thus $S$ is compact in $H_{l o c}^{-1}\left(\Omega^{\prime}\right)$. From (3.16) and (3.18)-(3.20), we have

$$
\left\|A_{1}+R+B+\Sigma\right\|_{\left(C_{0}\right)^{*}} \leq C .
$$

By the embedding theorem, $\left(C_{0}\left(\Omega^{\prime}\right)\right)^{*} \hookrightarrow W^{-1, p_{0}}$ is compact for $1<p_{0}<2$. Thus, $A_{1}+R+B+\Sigma$ is compact in $W^{-1, p_{0}}\left(\Omega^{\prime}\right)$. By the Sobolev theorem, $W_{0}^{1, p_{1}}\left(\Omega^{\prime}\right) \hookrightarrow$ $C_{0}^{\alpha}\left(\Omega^{\prime}\right), p_{1}>\frac{2}{1-\alpha}$ and the estimate

$$
\left|A_{2}(\varphi)\right| \leq C l^{\alpha-\frac{1}{2}}\|\varphi\|_{C_{0}^{\alpha}},
$$

we have

$$
\left|A_{2}(\varphi)\right| \leq C l^{\alpha-\frac{1}{2}}\|\varphi\|_{W_{0}^{1, p_{1}}\left(\Omega^{\prime}\right)} .
$$

It follows from duality that

$$
\left\|A_{2}\right\|_{W^{-1, p_{2}\left(\Omega^{\prime}\right)}} \leq C l^{\alpha-\frac{1}{2}} \rightarrow 0 \quad \text { as } \quad l \rightarrow 0 \quad \text { for } \quad 1<p_{2}<\frac{2}{1+\alpha}<2 .
$$

Then, $A_{2}$ is compact in $W^{-1, p_{2}}\left(\Omega^{\prime}\right)$. So $A+R+B+\Sigma$ is compact in $W^{-1, p}\left(\Omega^{\prime}\right)$, where $1<p<\min \left(p_{0}, p_{2}\right)<2$.

Next, from the uniform boundedness of $V^{l}$, we have the following fact:

$$
\eta\left(V^{l}\right)_{t}+q\left(V^{l}\right)_{x}-S \text { is bounded in } W^{-1, \infty}\left(\Omega^{\prime}\right) .
$$

Since $\Omega^{\prime}$ is bounded, the above statement implies that

$$
\eta\left(V^{l}\right)_{t}+q\left(V^{l}\right)_{x}-S \text { is bounded in } W^{-1, r}\left(\Omega^{\prime}\right), \quad \text { for } \quad r>1 .
$$

That is, $A+R+B+\Sigma$ is bounded in $W^{-1, r}\left(\Omega^{\prime}\right), r>1$. It follows from Lemma 3.4, that

$$
A+R+B+\Sigma \text { is compact in } H_{l o c}^{-1}\left(\Omega^{\prime}\right) .
$$

So

$$
\eta\left(V^{l}\right)_{t}+q\left(V^{l}\right)_{x}-S \text { is compact in } H_{l o c}^{-1}\left(\Omega^{\prime}\right)
$$


Since $S$ is also compact in $H_{l o c}^{-1}\left(\Omega^{\prime}\right)$, we conclude that $\eta\left(V^{l}\right)_{t}+q\left(V^{l}\right)_{x}$ is compact in $H_{l o c}^{-1}\left(\Omega^{\prime}\right)$.

Therefore, Theorem 3.1 follows from Theorem 3.2 and Theorem 3.3 .

3.3. Existence of weak solution. By Theorem 3.1, we obtain that as $l \rightarrow 0$ the approximate solutions $V^{l}(x, t) \rightarrow V(x, t)=(\rho(x, t), m(x, t))$ almost everywhere, and $I^{l}(\nu, \Omega, x, t)$ $\rightarrow I(\nu, \Omega, x, t)$ weakly-* in $\mathbb{R}_{+}^{2}$ for each fixed pair of parameters $(\nu, \Omega)$. Now, we will show that $(V, I)$ is a weak entropy solution of (2.1)-(2.2). We first recall the following useful lemma (cf. [7, 20]):

Lemma 3.5. Consider the following Riemann problem:

$$
\left\{\begin{array}{l}
\rho_{t}+m_{x}=0 \\
m_{t}+\left(\frac{m^{2}}{\rho}+P_{m}(\rho)\right)_{x}=0 \\
\left.(\rho, m)\right|_{t=0}= \begin{cases}\left(\rho_{l}, m_{l}\right), & x<0 \\
\left(\rho_{r}, m_{r}\right), & x>0\end{cases}
\end{array}\right.
$$

where $\rho_{l}, m_{l}, \rho_{r}, m_{r}$ are constants satisfying $0 \leq \rho_{l}, \rho_{r},\left|\frac{m_{l}}{\rho_{l}}\right|,\left|\frac{m_{r}}{\rho_{r}}\right|<\infty$. For any $\epsilon>0$, there exist constants $l>0$ and $h>0$ such that the solution of (3.23) in the region $\{(x, t):|x|<l, 0 \leq t<h\}$ satisfies

$$
\int_{-l}^{l}|\rho(x, t)-\rho(x, 0)| d x \leq C l \epsilon, \quad 0 \leq t \leq h,
$$

where $C$ depends only on the bounds of $\rho$ and $|m|$, and the mesh lengths $l$ and $h$ satisfy

$$
\max _{i=1,2}\left(\sup _{0 \leq t \leq h}\left|\lambda_{i}(\rho, m)\right|\right)<\frac{l}{2 h} .
$$

Theorem 3.4. Suppose that the conditions of (MA) are satisfied. Then the bounded measurable function $(V, I)$ is a weak entropy solution of (2.1)-(2.2); i.e., $(V, I)$ satisfies (2.3)-(2.5) and (2.11).

Proof. (1) For every test function $\varphi \in C_{0}^{\infty}\left(R_{T}\right)$ satisfying $\varphi(x, T)=0$, we consider the following integral identity:

$$
\int_{0}^{T} \int_{\mathbb{R}}\left(\frac{1}{c} I^{l} \varphi_{t}+\Omega_{1} I^{l} \varphi_{x}\right) d x d t+\int_{\mathbb{R}} \frac{1}{c} I^{l}(x, 0) \varphi(x, 0) d x+\int_{0}^{T} \int_{\mathbb{R}} F\left(\rho^{l}, I^{l}\right) \varphi d x d t=A(\varphi)+R(\varphi),
$$

where

$$
\begin{gathered}
A(\varphi)=\sum_{i, j} \frac{1}{c} \int_{(j-1) l}^{j l}\left(\left(I_{0}^{l}\right)^{\underline{i}}-\left(I^{l}\right)^{\underline{i}}\right) \varphi^{i} d x+\sum_{i, j} \iint F\left(\rho_{0}^{l}, I_{0}^{l}\right) \varphi d x d t \\
R(\varphi)=\sum_{i, j} \iint\left(\frac{1}{c}\left(I^{l}-I_{0}^{l}\right) \varphi_{t}+\Omega_{1}\left(I^{l}-I_{0}^{l}\right) \varphi_{x}\right) d x d t \\
+\sum_{i, j} \iint_{(j-1) l}^{j l}\left(F\left(\rho^{l}, I^{l}\right)-F\left(\rho_{0}^{l}, I_{0}^{l}\right)\right) \varphi d x d t .
\end{gathered}
$$


First for $R(\varphi)$, by (2.8) we have

$$
\begin{aligned}
R(\varphi) \leq & C h \sum_{i, j} \iint_{(j-1) l}^{j l}\left(\left|\varphi_{t}\right|+\left|\varphi_{x}\right|\right)\left|F\left(\rho_{0}^{l}, I_{0}^{l}\right)\right| d x d t \\
& +C\left(I^{l}, I_{0}^{l}\right) \sum_{i, j} \iint_{(j-1) l}^{j l}\left|\rho^{l}-\rho_{0}^{l}\right| \varphi d x d t \\
\leq & C h \sum_{i, j} \iint_{(j-1) l}^{j l}\left(\left|\varphi_{t}\right|+\left|\varphi_{x}\right|\right)\left|F\left(\rho_{0}^{l}, I_{0}^{l}\right)\right| d x d t \\
& +C h \sum_{i, j} \iint_{(j-1) l}^{j l}\left|G\left(\rho_{0}^{l}, I_{0}^{l}\right)\right| \varphi d x d t \\
\leq & C h\|\varphi\|_{C^{1}} \leq C l\|\varphi\|_{C^{1}} \rightarrow 0 \text { as } \quad l \rightarrow 0 .
\end{aligned}
$$

To estimate $A(\varphi)$, we decompose $A(\varphi)$ into three parts:

$$
\begin{aligned}
A(\varphi)= & \sum_{i, j} \frac{1}{c} \int_{(j-1) l}^{j l}\left(\left(I_{0}^{l}\right)^{\underline{i}}-\left(I^{l}\right)^{\underline{i}}\right)\left(\varphi^{i}-\varphi_{j}^{i}\right) d x \\
& +\sum_{i, j} \int_{i h}^{(i+1) h} \int F\left(\rho_{0}^{l}, I_{0}^{l}\right)\left(\varphi-\varphi_{j}^{i}\right) d x d t \\
& +\sum_{i, j} \int_{i h}^{(i+1) h} \int\left(F\left(\rho_{0}^{l}, I_{0}^{l}\right)-F\left(\left(\rho_{0}^{l}\right)^{\underline{i}},\left(I_{0}^{l}\right)^{\underline{i}}\right)\right) \varphi_{j}^{i} d x d t \\
\equiv & A_{1}(\varphi)+A_{2}(\varphi)+A_{3}(\varphi) .
\end{aligned}
$$

For $A_{1}(\varphi)$, we have

$$
\begin{aligned}
\left|A_{1}(\varphi)\right| & =\frac{1}{c}\left|\sum_{i, j} \int F\left(\left(\rho_{0}^{l}\right)^{\underline{i}},\left(I_{0}^{l}\right)^{\underline{i}}\right) h\left(\varphi^{i}-\varphi_{j}^{i}\right) d x\right| \\
& \leq C h\left(\sum_{i, j} \int F^{2}\left(\left(\rho_{0}^{l}\right)^{\underline{i}},\left(I_{0}^{l}\right)^{i}\right) d x\right)^{\frac{1}{2}}\left(\sum_{i, j} \int_{(j-1) l}^{j l}\left(\varphi^{i}-\varphi_{j}^{i}\right)^{2} d x\right)^{\frac{1}{2}} \\
& \leq C T\left\|F^{2}\left(\rho_{0}^{l}, I_{0}^{l}\right)\right\|_{L^{\infty}}^{1 / 2} \times l^{1 / 2}\|\varphi\|_{C^{1}} \\
& \leq C l^{1 / 2}\|\varphi\|_{C^{1}} \rightarrow 0 \text { as } \quad l \rightarrow 0,
\end{aligned}
$$

and for $A_{2}(\varphi)$, one has

$$
\begin{aligned}
\left|A_{2}(\varphi)\right| & \leq \sum_{i, j} \iint_{(j-1) l}^{j l}\left|F\left(\rho_{0}^{l}, I_{0}^{l}\right)\right|\left(\frac{\left|\varphi^{i}-\varphi_{j}^{i}\right|}{|x-j l|} l+\frac{\left|\varphi-\varphi^{i}\right|}{|t-i h|} h\right) d x d t \\
& \leq C l\|F\|_{L^{\infty}\|\varphi\|_{C^{1}}} \\
& \leq C l\|\varphi\|_{C^{1}} \rightarrow 0 \text { as } \quad l \rightarrow 0 .
\end{aligned}
$$


By (2.8) and Lemma 3.5, we have

$$
\begin{aligned}
\left|A_{3}(\varphi)\right| & =\left|\sum_{i, j} \int_{i h}^{(i+1) h} \int\left(F\left(\rho_{0}^{l}, I_{0}^{l}\right)-F\left(\left(\rho_{0}^{l}\right)^{\underline{i}},\left(I_{0}^{l}\right)^{\underline{i}}\right)\right) \varphi_{j}^{i} d x d t\right| \\
& \leq C\left(I_{0}^{l},\left(I_{0}^{l}\right)^{\underline{i}}\right)\|\varphi\|_{L^{\infty}} \sum_{i, j} \iint_{(j-1) l}^{j l}\left|\rho_{0}^{l}-\left(\rho_{0}^{l}\right)^{\underline{i}}\right| d x d t \\
& \leq C\|\varphi\|_{L^{\infty}} \sum_{i} \int_{i h}^{(i+1) h} \epsilon d t \\
& \leq C \epsilon\|\varphi\|_{L^{\infty}},
\end{aligned}
$$

where $\epsilon>0$ is an arbitrarily small constant. It follows from (3.24)-(3.27) and the assumption (2.8) that, as $l \rightarrow 0$,

$$
\int_{0}^{T} \int_{\mathbb{R}}\left(\frac{1}{c} I \varphi_{t}+\Omega_{1} I \varphi_{x}\right) d x d t+\int_{\mathbb{R}} \frac{1}{c} I_{0} \varphi(x, 0) d x+\int_{0}^{T} \int_{\mathbb{R}} F(\rho, I) \varphi d x d t=0 .
$$

(2) For every test function $\varphi \in C_{0}^{\infty}\left(R_{T}\right)$ satisfying $\varphi(x, T)=0$, we consider the following integral identity:

$$
\int_{0}^{T} \int_{\mathbb{R}}\left(\rho^{l} \varphi_{t}+m^{l} \varphi_{x}\right) d x d t+\int_{\mathbb{R}} \rho^{l}(x, 0) \varphi(x, 0) d x=A(\varphi)+R(\varphi),
$$

where

$$
\begin{aligned}
& A(\varphi)=\sum_{i, j} \int_{(j-1) l}^{j l}\left(\left(\rho^{l}\right)^{i}-\rho_{j}^{i}\right) \varphi^{i} d x, \\
& R(\varphi)=\sum_{i, j} \int_{i h}^{(i+1) h} \int_{\mathbb{R}}\left(m^{l}-m_{0}^{l}\right) \varphi_{x} d x d t .
\end{aligned}
$$

We claim that $A(\varphi), R(\varphi) \rightarrow 0$, as $l \rightarrow 0$. For $A(\varphi)$, one has

$$
\begin{aligned}
|A(\varphi)| & =\left|\sum_{i, j} \int_{(j-1) l}^{j l}\left(\left(\rho^{l}\right)^{\underline{i}}-\rho_{j}^{i}\right)\left(\varphi^{i}-\varphi_{j}^{i}\right) d x\right| \\
& \left.\leq\left.\left(\sum_{i, j} \int_{(j-1) l}^{j l}\left|\varphi^{i}-\varphi_{j}^{i}\right|^{2} d x\right)^{\frac{1}{2}}\left(\sum_{i, j} \int_{(j-1) l}^{j l} \mid\left(\rho^{l}\right)^{\underline{i}}-\rho_{j}^{i}\right)\right|^{2} d x\right)^{\frac{1}{2}} \\
& \left.\leq\left. C\|\varphi\|_{C^{1}}\left(\sum_{i, j} \int_{(j-1) l}^{j l}|x-j l|^{2} d x\right)^{\frac{1}{2}}\left(\sum_{i, j} \int_{(j-1) l}^{j l} \mid\left(\rho^{l}\right)^{\underline{i}}-\rho_{j}^{i}\right)\right|^{2} d x\right)^{\frac{1}{2}} \\
& \leq C l^{1 / 2}\|\varphi\|_{C^{1}} \rightarrow 0 \quad \text { as } \quad l \rightarrow 0 .
\end{aligned}
$$


It follows from the uniform bound for $\left(V^{l}, I^{l}\right)$ that

$$
\begin{aligned}
|R(\varphi)| & =\sum_{i, j} \int_{i h}^{(i+1) h} \int_{\mathbb{R}} G\left(\rho_{0}^{l}, I_{0}^{l}\right) \varphi_{x}(t-i h) d x d t \\
& \leq C l\left\|G\left(\rho_{0}^{l}, I_{0}^{l}\right)\right\|_{L^{\infty}} \int_{0}^{T} \int_{\Omega^{\prime}}\left|\varphi_{x}\right| d x d t \\
& \leq C l\|\varphi\|_{C^{1}} \rightarrow 0 \quad \text { as } \quad l \rightarrow 0 .
\end{aligned}
$$

Then (3.28)-(3.29) imply that

$$
\lim _{l \rightarrow 0} \int_{0}^{T} \int_{\mathbb{R}}\left(\rho^{l} \varphi_{t}+m^{l} \varphi_{x}\right) d x d t+\int_{\mathbb{R}} \rho^{l}(x, 0) \varphi(x, 0) d x=0 .
$$

Applying the dominated convergence theorem to (3.30), we have

$$
\int_{0}^{T} \int_{\mathbb{R}}\left(\rho \varphi_{t}+m \varphi_{x}\right) d x d t+\int_{\mathbb{R}} \rho_{0} \varphi(x, 0) d x=0 .
$$

(3) For every test function $\varphi \in C_{0}^{\infty}\left(R_{T}\right)$ satisfying $\varphi(x, T)=0$, we consider the following integral identity:

$$
\int_{0}^{T} \int_{\mathbb{R}}\left(m^{l} \varphi_{t}+f\left(V^{l}\right) \varphi_{x}+G\left(\rho^{l}, I^{l}\right) \varphi\right) d x d t+\int_{\mathbb{R}} m^{l}(x, 0) \varphi(x, 0) d x=A(\varphi)+R(\varphi),
$$

where $f(V)=\frac{m^{2}}{\rho}+\frac{\rho^{\gamma}}{\gamma}$, and

$$
\begin{gathered}
A(\varphi)=\sum_{i, j} \int\left(\left(m_{0}^{l}\right)^{-}-m_{j}^{i}\right) \varphi^{i} d x+\sum_{i, j} \iint G\left(\rho_{0}^{l}, I_{0}^{l}\right) \varphi d x d t \\
R(\varphi)=\sum_{i, j} \iint\left(\left(m^{l}-m_{0}^{l}\right) \varphi_{t}+\left(f\left(V^{l}\right)-f\left(V_{0}^{l}\right)\right) \varphi_{x}+\left(G\left(\rho^{l}, I^{l}\right)-G\left(\rho_{0}^{l}, I_{0}^{l}\right)\right) \varphi\right) d x d t .
\end{gathered}
$$

We estimate $R(\varphi)$ first. By (2.9), we have

$$
\begin{aligned}
|R(\varphi)| \leq & h \sum_{i, j} \iint_{(j-1) l}^{j l}\left|G\left(\rho_{0}^{l}, I_{0}^{l}\right) \| \varphi_{t}\right| d x d t \\
& +h \sum_{i, j} \iint_{(j-1) l}^{j l}\left|\nabla f\left(\xi_{j}^{i}\right) \| G\left(\rho_{0}^{l}, I_{0}^{l}\right)\right|\left|\varphi_{x}\right| d x d t \\
& +h \sum_{i, j} \iint_{(j-1) l}^{j l}\left|F\left(\rho_{0}^{l}, I_{0}^{l}\right) \| \varphi\right| d x d t \\
\leq & C l\|\varphi\|_{C^{1}} \rightarrow 0 \quad \text { as } \quad l \rightarrow 0,
\end{aligned}
$$

where $\xi_{j}^{i}$ is the mean value of $V^{l}$ on the region $((j-1) l, j l) \times(i h,(i+1) h)$. 
We decompose $A(\varphi)$ into three parts:

$$
\begin{aligned}
A(\varphi)= & \sum_{i, j} \int_{(j-1) l}^{j l}\left(\left(m_{0}^{l}\right)^{\underline{i}}-m_{j}^{i}\right)\left(\varphi^{i}-\varphi_{j}^{i}\right) d x \\
& +\sum_{i, j} \int_{i h}^{(i+1) h} \int G\left(\rho_{0}^{l}, I_{0}^{l}\right)\left(\varphi-\varphi_{j}^{i}\right) d x d t \\
& +\sum_{i, j} \int_{i h}^{(i+1) h} \int\left(G\left(\rho_{0}^{l}, I_{0}^{l}\right)-G\left(\left(\rho_{0}^{l}\right)^{\underline{i}},\left(I_{0}^{l}\right)^{\underline{i}}\right)\right) \varphi_{j}^{i} d x d t \\
\equiv & A_{1}(\varphi)+A_{2}(\varphi)+A_{3}(\varphi) .
\end{aligned}
$$

For $A_{1}(\varphi)$, using Lemma 3.1 , we have

$$
\begin{aligned}
&\left|A_{1}(\varphi)\right|=\left|\sum_{i, j} \int\left(h G\left(\left(\rho_{0}^{l}\right)^{-},\left(I_{0}^{l}\right)^{\underline{i}}\right)+\left(m^{l}\right)^{\underline{i}}-m_{j}^{i}\right)\left(\varphi^{i}-\varphi_{j}^{i}\right) d x\right| \\
& \leq\left(\sum_{i, j} \int_{(j-1) l}^{j l}\left(\varphi^{i}-\varphi_{j}^{i}\right)^{2} d x\right)^{\frac{1}{2}}\left[h\left(\sum_{i, j} \int G^{2}\left(\left(\rho_{0}^{l}\right)^{\underline{i}},\left(I_{0}^{l}\right)^{\prime}\right) d x\right)^{\frac{1}{2}}\right. \\
&\left.+\left(\sum_{i, j} \int_{(j-1) l}^{j l}\left(\left(m^{l}\right)^{\underline{i}}-m_{j}^{i}\right)^{2} d x\right)^{\frac{1}{2}}\right] \\
& \leq C l^{1 / 2}\|\varphi\|_{C^{1}}\left(T\left\|G^{2}\left(\rho_{0}^{l}, I_{0}^{l}\right)\right\|_{L^{\infty}}+C\right) \leq C l^{1 / 2}\|\varphi\|_{C^{1}} \rightarrow 0 \text { as } \quad l \rightarrow 0,
\end{aligned}
$$

and for $A_{2}(\varphi)$, one has

$$
\begin{aligned}
\left|A_{2}(\varphi)\right| & \leq \sum_{i, j} \iint_{(j-1) l}^{j l}\left|G\left(\rho_{0}^{l}, I_{0}^{l}\right)\right|\left(\frac{\left|\varphi^{i}-\varphi_{j}^{i}\right|}{|x-j l|} l+\frac{\left|\varphi-\varphi^{i}\right|}{|t-i h|} h\right) d x d t \\
& \leq C l\|G\|_{L^{\infty}}\|\varphi\|_{C^{1}} \leq C l\|\varphi\|_{C^{1}} \rightarrow 0 \quad \text { as } \quad l \rightarrow 0 .
\end{aligned}
$$

By (2.9) and Lemma 3.5, we have

$$
\begin{aligned}
\left|A_{3}(\varphi)\right| & \leq C\left|\sum_{i, j} \int_{i h}^{(i+1) h} \int\left(G\left(\rho_{0}^{l}, I_{0}^{l}\right)-G\left(\left(\rho_{0}^{l}\right)^{\underline{i}},\left(I_{0}^{l}\right)^{i}\right)\right) \varphi_{j}^{i} d x d t\right| \\
& \leq C\left(I_{0}^{l},\left(I_{0}^{l}\right)^{\underline{i}}\right)\|\varphi\|_{L^{\infty}} \sum_{i, j} \iint_{(j-1) l}^{j l}\left|\rho_{0}^{l}-\left(\rho_{0}^{l}\right)^{i}\right| d x d t \\
& \leq C\|\varphi\|_{L^{\infty}} \sum_{i} \int_{i h}^{(i+1) h} \epsilon d t \\
& \leq C \epsilon\|\varphi\|_{L^{\infty}}
\end{aligned}
$$

where $\epsilon>0$ is an arbitrarily small constant.

It follows from (3.31)-(3.34) that

$$
\lim _{l \rightarrow 0} \int_{0}^{T} \int_{\mathbb{R}}\left(m^{l} \varphi_{t}+f\left(V^{l}\right) \varphi_{x}+G\left(\rho^{l}, I^{l}\right) \varphi\right) d x d t+\int_{\mathbb{R}} m^{l}(x, 0) \varphi(x, 0) d x=0 .
$$


Using the dominated convergence theorem, we have

$$
\int_{0}^{T} \int_{\mathbb{R}}\left(m \varphi_{t}+\left(\frac{m^{2}}{\rho}+P_{m}(\rho)\right) \varphi_{x}\right) d x d t+\int_{0}^{T} \int_{\mathbb{R}} G(\rho, I) \varphi d x+\int_{\mathbb{R}} m_{0} \varphi(x, 0) d x=0 .
$$

(4) For every weak and convex entropy pair $(\eta, q)$ and every nonnegative smooth function $\psi$ that has compact support in region $R_{T}$ satisfying $\psi(x, 0)=\psi(x, T)=0$, we consider the following integral identity:

$$
\int_{0}^{T} \int_{\mathbb{R}}\left(\eta\left(V^{l}\right) \psi_{t}+q\left(V^{l}\right) \psi_{x}\right) d x d t=A(\psi)+R(\psi)+\Sigma(\psi)+S(\psi)
$$

where $A(\psi), R(\psi), \Sigma(\psi), S(\psi)$ are similar to those of (3.15). Since $(\eta, q)$ is a convex entropy pair and $\psi \geq 0$, we have

$$
\begin{aligned}
& \Sigma(\psi) \geq 0 \\
& A(\psi)=\left.\sum_{i, j} \psi_{j}^{i} \int_{(j-1) l}^{j l}\left(\left(V^{l}\right)^{\underline{i}}-V_{j}^{i}\right)^{\top} \nabla^{2} \eta\left(\xi_{j}^{i}\right)\left(\left(V^{l}\right)^{\underline{i}}-V_{j}^{i}\right)\right) d x \\
&+\sum_{i, j} \int_{(j-1) l}^{j l}\left(\eta\left(V^{l}\right)^{\underline{i}}-\eta\left(V_{j}^{i}\right)\right)\left(\psi^{i}-\psi_{j}^{i}\right) d x \\
& \geq \sum_{i, j} \int_{(j-1) l}^{j l}\left(\eta\left(V^{l}\right)^{\underline{i}}-\eta\left(V_{j}^{i}\right)\right)\left(\psi^{i}-\psi_{j}^{i}\right) d x \\
& \geq-C l^{\alpha-1 / 2}\|\psi\|_{C_{0}^{\alpha}}, \quad \frac{1}{2}<\alpha<1 .
\end{aligned}
$$

As in (3.21), we have

$$
\begin{aligned}
& S(\psi) \geq-C l\|\psi\|_{H_{0}^{1}}, \\
& R(\psi)=-h \sum_{i, j} \int\left(\int_{0}^{1} \eta_{m}\left(\left(V^{l}\right)^{\underline{i}}+\theta\left(\left(V_{0}^{l}\right)^{\underline{i}}-\left(V^{l}\right)^{\underline{i}}\right)\right) d \theta\right) G\left(\left(\rho_{0}^{l}\right)^{\underline{i}},\left(I_{0}^{l}\right)^{\underline{i}}\right) \psi^{i} d x \\
& =-h \sum_{i, j} \int\left(\int_{0}^{1} \eta_{m}\left(\left(V^{l}\right)^{\underline{i}}+\theta\left(\left(V_{0}^{l}\right)^{\underline{i}}-\left(V^{l}\right)^{\underline{i}}\right)\right) d \theta\right) \\
& \times\left(G\left(\left(\rho_{0}^{l}\right)^{\underline{i}},\left(I_{0}^{l}\right)^{\underline{\underline{i}}}\right)-G\left(\left(\rho^{l}\right)^{\underline{i}},\left(I^{l}\right)^{\underline{i}}\right)\right) \psi^{i} d x \\
& -h \sum_{i, j} \int\left(\int_{0}^{1} \eta_{m}\left(\left(V^{l}\right)^{\underline{i}}+\theta\left(\left(V_{0}^{l}\right)^{\underline{i}}-\left(V^{l}\right)^{\underline{i}}\right)\right) d \theta\right) G\left(\left(\rho^{l}\right)^{\underline{i}},\left(I^{l}\right)^{\underline{i}}\right) \psi^{i} d x \\
& \geq-C l-h \sum_{i, j} \int\left(\int_{0}^{1} \eta_{m}\left(\left(V^{l}\right)^{\underline{i}}+\theta\left(\left(V_{0}^{l}\right)^{\underline{i}}-\left(V^{l}\right)^{\underline{i}}\right)\right) d \theta\right) G\left(\left(\rho^{l}\right)^{\underline{i}},\left(I^{l}\right)^{\underline{i}}\right) \psi^{i} d x .
\end{aligned}
$$


It follows from (3.35)-(3.38) that

$$
\begin{aligned}
& \int_{0}^{T} \int_{\mathbb{R}}\left(\eta\left(V^{l}\right) \psi_{t}+q\left(V^{l}\right) \psi_{x}\right) d x d t \\
& \quad+h \sum_{i, j} \int\left(\int_{0}^{1} \eta_{m}\left(\left(V^{l}\right)^{\underline{i}}+\theta\left(\left(V_{0}^{l}\right)^{\underline{i}}-\left(V^{l}\right)^{\underline{i}}\right)\right) d \theta\right) G\left(\left(\rho^{l}\right)^{\underline{i}},\left(I^{l}\right)^{\underline{i}}\right) \psi^{i} d x \\
& \geq-C\left(\|\psi\|_{C_{0}^{\alpha}}+l^{3 / 2-\alpha}\left(1+\|\psi\|_{H_{0}^{1}}\right)\right) l^{\alpha-1 / 2}, \quad \frac{1}{2}<\alpha<1 .
\end{aligned}
$$

Letting $l \rightarrow 0$ in (3.39) and using the fact that $V^{l} \rightarrow V$ a.e. and $I^{l} \rightarrow I$ weakly-* in $\mathbb{R}_{+}^{2}$ for each fixed pair of parameters $(\nu, \Omega)$, we obtain the following entropy condition:

$$
\int_{0}^{T} \int_{\mathbb{R}}\left(\eta(\rho, m) \psi_{t}+q(\rho, m) \psi_{x}\right) d x d t+\int_{0}^{T} \int_{\mathbb{R}} \eta_{m}(\rho, m) G(\rho, I) \psi d x d t \geq 0 .
$$

This completes the proof of the main result in Theorem 2.1.

Acknowledgement. The paper was completed during Peng Jiang's visiting the University of Pittsburgh. Peng Jiang's research was supported in part by a joint project from the NSAF of China under the grant 10676020, and a key project of NSFC under the grant 10531020. Dehua Wang's research was supported in part by the National Science Foundation under grants DMS-0604362 and DMS-0906160, and by the Office of Naval Research under grant N00014-07-1-0668.

\section{REFERENCES}

[1] C. Buet, B. Despres, Asymptotic analysis of fluid models for coupling of radiation and hydrodynamics, J. of Quantitative Spectroscopy and Radiative Transfer. 85 (2004), 385-418.

[2] G.-Q. Chen, Convergence of Lax-Friedrichs scheme for isentropic gas dynamics. III, Acta Math. Sci. 6 (1986), 75-120. MR.924671 (89f:76007)

[3] G.-Q. Chen, T.-H. Li, Global entropy solution in $L^{\infty}$ to the Euler equations and Euler-Poisson equations for isothermal fluid with spherical symmetry, Methods and Applications of Analysis. Vol. 10, No. 2 (2003), 215-244. MR2074749 (2005f:35255)

[4] G.-Q. Chen, D. Wang, Convergence of shock capturing scheme for the compressible Euler-Poisson equations, Commun. Math. Phys. 179 (1996), 333-364. MR1400743 (97j:35119)

[5] G.-Q. Chen, D. Wang, The Cauchy problem for the Euler equations for compressible ßuids, Handbook of Mathematical Fluid Dynamics, 421-543, Amsterdam: North-Holland, 2002. MR1942468 (2004e:35182)

[6] X. Ding, G.-Q. Chen, P. Lou, Convergence of the Lax-Friedrichs scheme for isentropic gas dynamics, I, II, Acta Math. Sci. 5 (1985), 415-432, 433-472. MR.922139 (89f:76006)

[7] X. Ding, G.-Q. Chen, P. Lou, Convergence of the fraction step Lax-Friedrichs scheme and Godunov scheme for the isentropic system of gas dynamics, Commun. Math. Phys. 121 (1989), 63-84. MR985615 (90d:65168)

[8] R. J. DiPerna, Convergence of the viscosity method for isentropic gas dynamics, Commun. Math. Phys. 91 (1983), 1-30. MR719807(85i:35118)

[9] R. J. DiPerna, Convergence of approximate solutions to conservation laws, Arch. Rat. Mech. Anal. 82 (1983), 27-70. MR684413 (84k:35091)

[10] P. Jiang, D. Wang, Formation of singularities of solutions to the three-dimensional Euler-Boltzmann equations in radiation hydrodynamics, Nonlinearity 23 (2010), 809-821. MR 2602015 (2011c:76093)

[11] S. Jiang, X. H. Zhong, Local existence and finite-time blow-up in multidimensional radiation hydrodynamics, J. Math. Fluid. Mech. 9 (2007), 543-564. MR.2374158(2009h:76232) 
[12] S. Kawashima, S. Nishibata, Cauchy problem for a model system of radiating gas: weak solution with a jump and classical solutions, Math. Model Mech. Appl. Sci. 9 (1999), 69-91. MR1671523 (99m:35151)

[13] S. Kawashima, S. Nishibata, Shock wave for a model system of the radiation gas, SIAM. J. Math. Anal. 30, No. 1 (1999), 95-117. MR.1646685 (99h:35133)

[14] P.-L. Lions, B. Perthame, E. Souganidis, Existence and stability of entropy solutions for the hyperbolic systems of isentropic gas dynamics in Eulerian and Lagrangian coordinates, Comm. Pure Appl. Math. 49 (1996), 599-638. MR.1383202 (97e:35107)

[15] P.-L. Lions, B. Perthame, E. Tadmor, Kinetic formulation of the isentropic gas dynamics and p-systems, Commun. Math. Phys. 163 (1994), 415-431. MR.1284790 (95c:76093)

[16] T. Makino, S. Takeno, Initial boundary value problem for the spherically symmetric motion of isentropic gas, Japan J. Indust. Appl. Math. 11 (1994), 171-183. MR.1266527 (95h:76095)

[17] O. Mihalas, B. W. Mihalas, Foundations of Radiation Hydrodynamics, Oxford Univ. Press, New York, Oxford, 1984. MR781346 (86h:85004)

[18] G. C. Pomraning, The Equation of Radiation Hydrodynamics, Pergamon Press, 1973.

[19] D. Wang, Global solutions to the Euler-Poisson equations of two-carrier types in one dimension, Z. angew. Math. Phys. 48 (1997), 680-693. MR1471476 (98f:35101)

[20] B. Zhang, Convergence of the Godunov scheme for a simplified one-dimensional hydrodynamic model for semiconductor devices, Commun. Math. Phys. 157 (1993), 1-22. MR1244856 (95e:82080) 